\title{
A experiência de uso com materiais no design de produto: Uma revisão integrativa sobre a percepção do usuário
}

\author{
Mariana Araujo Laranjeira; \\ Luis Carlos Paschoarelli; \\ Marizilda dos Santos Menezes
}

resumo:

A experiência do usuário no design de produtos está diretamente relacionada com a perceção dos materiais que envolvem e consolidam o artefato. Este modo de interação envolve questões que vão desde a forma e as propriedades físicas do mesmo, até a implicação de aspectos pessoais e sociais do usuário. A experiência com materiais explora as características estéticas, sensoriais e do contexto de uso, contribuindo para que o designer entenda melhor o usuário e desenvolva produtos com design mais empático e hedônico. Isso pode trazer contribuições também na experimentação com novos materiais e na emersão de questionamentos associados à sustentabilidade dos recursos. Nesse contexto, esta investigação teve por objetivo entender como se dá a experiência do usuário com materiais. Por meio de uma revisão bibliográfica sistemática, a pesquisa integrou 15 estudos da área do design sobre práticas de experimentação específicas com materiais. Os resultados da análise permitiram identificar os tipos de estímulos utilizados, as características dos materiais envolvidos e as qualidades cognitivas da experiência do usuário.

\section{palavras-chave:}

Design; experiência do usuário; ergonomia; percepção; materiais; 


\section{Introdução}

Muito além de cumprir seus requisitos de funcionalidade, um produto deve ser capaz de fornecer experiências a seus usuários (ALLAM et al., 2013). Dentro de um contexto de uso específico, "a experiência do usuário é a soma das emoções, percepções e reações que uma pessoa experimenta ao interagir com um produto ou serviço" (TOSI, 2020, p.51), o que caracteriza uma combinação de usabilidade, utilidade e impacto emocional.

Nesse contexto, os artefatos carregam um significado expressivo vinculado à percepção humana e os materiais que os compõem são considerados um dos principais veículos em proporcionar experiências variadas, sejam elas sensoriais ou emocionais (MONNA, 2017). As diversas propriedades físicas e ópticas dos materiais determinam o modo como se dá a interação com eles, desde o primeiro contato até o uso do produto em si (NIU; LO, 2019).

No design de produtos, os materiais são considerados parte fundamental para a estruturação dos artefatos físicos, pois fornecem à esses funcionalidade e sentido (VEELAERT et al., 2020). Dessa forma, a personalidade de um produto é diretamente influenciada pelos materiais que o compõem, e suas características incentivam a interação de seus usuários (CHOI, 2016).

Karana et al. (2015) afirmam que do ponto de vista da interação do usuário com o material é preciso observar a importância da percepção sensorial, ou seja, dos sentidos. Estes podem motivar reações positivas ou negativas nas relações usuário-produto, influenciando diretamente no ciclo de vida do mesmo e em preocupações com um design mais sustentável (NDENGUE et al. 2017).

Por uma perspectiva sensorial, a interação com materiais é um fenômeno complexo que deve considerar fatores contextuais e temporais relacionados ao produto, incorporando o contexto de uso e os aspectos socioculturais envolvidos (VEELAERT et al., 2020).

Durante a prática experimental, as experiências com materiais devem lidar com questões que relacionam todos os aspectos do objeto, como, por exemplo, suas propriedades físicas, até características do usuário e do processo de uso (KARANA et al., 2015). Nesse sentido, o design ergonômico está presente, entre outras coisas, em pesquisas que avaliam a interação do usuário com os aspectos físicos e cognitivos dos produtos acabados. Para se atingir as necessidades ergonômicas da interação entre o usuário e o produto, é preciso o cumprimento de questões relacionadas à segurança, funcionalidade, usabilidade e satisfação psicológica e sociológica do usuário (CYBIS et al., 2015). Assim, ao se identificar como os usuários percebem o material e como ocorre essa experiência, é possível estabelecer uma base de conhecimentos para o design de produtos mais ergonômicos, empáticos e hedônicos.

Novas abordagens no estudo de materiais dentro do contexto do design abrem perspectivas para investigações que exploram mais a fundo questões relacionadas com a experiência do usuário na interação com os materiais aplicados em produtos. Karana, Pedgley e Rognoli (2015) propõe o modelo do design orientado pelo material - Material Driven Design -, que busca incentivar o designer em explorar materiais novos e criar perspectivas sustentáveis, proporcionando experiências sensoriais, afetivas e emocionais aos usuários, por meio da interação física e perceptiva com materiais diferenciados. Com base nisso, Karana et al. (2015) afirmam que

[...] within the complexity inherent to materials and design-whether driven by technological or sustainability perspectives - users are the ones who determine the ultimate success (or not) of material choices. That is, success is a reflection of how people positively experience and react to the materials chosen by designers. (KARANA et al., 2015, p.16)

Veelaert et al. (2019) consideram que o desenvolvimento de pesquisas mais específicas aos materiais utilizados no design incentivam uma abordagem subjetiva orientada em identificar o papel desses na geração de significado nos produtos. Além disso, podem contribuir para a exploração de materiais inovadores ainda pouco explorados, em questões de novos processos de fabricação e uso, e na consolidação de uma nova estética material.

Levando esses conceitos em consideração, o objetivo principal deste trabalho foi entender como os usuários "criam" condições de experiências com materiais aplicados, em produtos variados, 
analisando questões de percepção e estabelecendo parâmetros para pesquisas sobre a interação entre humano $\mathrm{x}$ material $\mathrm{x}$ produto.

Neste sendito, o propósito deste estudo foi realizar uma revisão bibliográfica integrativa visando identificar como as diferentes pesquisas na área abordam a experiência dos usuários, particularmente em relação aos materiais aplicados no desenvolvimento de projeto de produtos; e como disponibilizam as informações pertinentes à atividade. Deste modo, o tipo de usuário ou a especificação do produto em si não foram considerados como relevantes, e o foco nessa situação específica se manteve exclusivamente nos diferentes protocolos de experimentação.

\section{Materiais e métodos}

Seguindo as diretrizes para o desenvolvimento de uma revisão sistemática integrativa, utilizou-se o método PRISMA (2020), que oferece um protocolo de registro e estruturação das informações, indicando as fases da coleta e um diagrama para os resultados obtidos.

De acordo com as etapas recomendadas, a revisão se constituiu da identificação dos artigos com os descritores definidos [1 - Identificação]; uma triagem por meio da exclusão de artigos não condizentes com a temática proposta [2 - Triagem]; a definição de critérios de inclusão para a elegibilidade dos artigos a serem lidos [3 - Elegibilidade]; e a seleção dos artigos finais a serem avaliados e integrados qualitativamente [4 - Inclusão].

Na primeira etapa de busca [1 - Identificação] utilizou-se como estratégia o método PICO (População, Interação, Comparação e Outcome/Resultado), que prevê a definição da questão de pesquisa juntamente com os descritores que serão empregados nos mecanismos de busca definidos para a investigação bibliográfica. Vale observar que para a questão estabelecida não se considerou o elemento PICO da Comparação. Assim, a investigação da literatura teve por objetivo identificar "Como se dá a experiência do usuário [População] na interação com materiais aplicados em produtos [Interação], considerando-se questões de percepção física, sensorial e cognitiva [Outcomes/Resultados]".

Os descritores foram definidos com base nesse questionamento e sofreram alteração e adaptação conforme a realização das buscas, pois a combinação e inclusão de palavras, assim como a manipulação de operadores boleanos (AND/OR), tem influência na aparição de resultados que, muitas vezes, podem ser em quantidades inviáveis para o desenvolvimento da revisão proposta. Desta forma, ficaram definidos, em inglês, os seguintes descritores:

Quadro 1: Descritores para os mecanismos de busca

\section{(User*) AND (Product) AND (Material) AND (Experience) AND/OR (Perception)}

Fonte: Elaborado pelos autores

Para a realização da pesquisa foram selecionados quatro mecanismos de busca que apresentassem publicações mais relacionadas com a área do design (Figura 1). Apesar da exibição dos artigos ter sido diferenciada em cada website, em todos eles foram utilizados os mesmos descritores definidos. Para gerenciar as referências encontradas utilizou-se o programa Mendeley Desktop e o Excel.

Figura 1: Bases de dados selecionadas

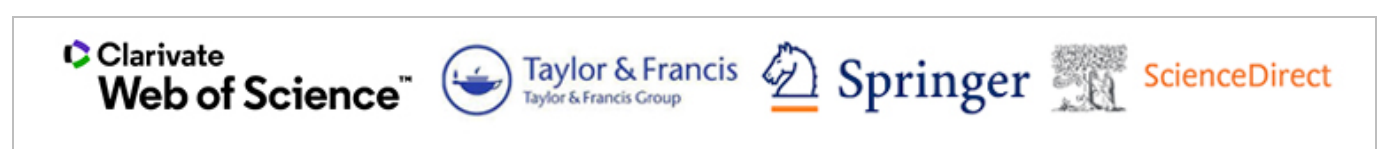

Fonte: Elaborado pelos autores

Devido à natureza predominantemente qualitativa dos artigos revisados, característica comum na área do design, é significativo informar que o intuito da revisão sistemática foi a integração e descrição de resultados e não uma meta-análise estatística dos dados. 
Para a segunda etapa do processo de coleta [2 - Triagem], definiram-se critérios de inclusão e exclusão das publicações (Quadro 2). Estes consideram o tipo de documento encontrado, o idioma da pesquisa, o escopo referente à temática do design e o período de publicação, filtrando-se artigos publicados entre 2015 e 2020 para uma avaliação mais atual do estado da arte de investigações dessa natureza experimental com materiais.

Quadro 2: Critérios de inclusão e exclusão da pesquisa

\begin{tabular}{|c|l|l|}
\hline $\begin{array}{c}\text { TIPO DE } \\
\text { DOCUMENTO }\end{array}$ & $\begin{array}{l}\text { CRITÉRIOS DE INCLUSÃO } \\
\text { Artigos científicos publicados em } \\
\text { periódicos internacionais e artigos } \\
\text { publicados em anais de } \\
\text { conferências/congressos. }\end{array}$ & $\begin{array}{l}\text { CRITÉRIOS DE EXCLUSÃO } \\
\text { Que não caracterizem artigos de periódicos e } \\
\text { artigos de conferências ou congressos. } \\
\text { (Exemplo: Livros, capitulos de livros, } \\
\text { resumos expandidos, patentes, entre outros) }\end{array}$ \\
\hline IDIOMA & Artigos escritos em inglês. & Artigos em idiomas diferentes do inglês. \\
\hline ANO & $\begin{array}{l}\text { Artigos publicados de 2015 até o } \\
\text { presente momento, 2020. }\end{array}$ & Trabalhos publicados anteriormente à 2015. \\
\hline ESCOPO & $\begin{array}{l}\text { Artigos que tratem concomitantemente } \\
\text { do design de produtos, a experiência do do } \\
\text { usuário e a experiência com materiais. }\end{array}$ & $\begin{array}{l}\text { Trabalhos que não atendam à temática da } \\
\text { revisão. Que estejam fora do contexto dos } \\
\text { materiais no design de produtos e que não } \\
\text { incluam algum tipo de percepção da } \\
\text { experiência do usuário. }\end{array}$ \\
\hline
\end{tabular}

Fonte: Elaborado pelos autors

Os artigos filtrados nessa etapa foram lidos com o intuito de se distinguir a assimilaridade com a temática da pesquisa [3 - Elegibilidade]. Uma primeira leva foi descartada com a leitura do Resumo/Abstract, e após a leitura completa das publicações restantes foram definidas aquelas publicações condizentes com os requisitos de interpretação e análise [4 - Inclusão].

Os critérios de análise se dividiram em duas categorias, sendo a primeira referente à qualidade do conteúdo do artigo como um todo (Quadro 3), e a segunda com relação às questões da experiência do usuário com o material (Quadro 4).

Para os primeiros critérios analisados consideraram-se questões associadas com ao rigor do trabalho de pesquisa desenvolvido, como por exemplo a definição dos objetivos, materiais, métodos e a obtenção de conclusões pertinentes. Também foi incluída uma última questão referente à preocupação dos autores com a sustentabilidade dos materiais do estudo.

Quadro 3: Critérios de avaliação de qualidade do artigo completo

\begin{tabular}{|l|l|l|c|}
\hline & QUESTÕES & RESPOSTA $=$ PONTUAÇÃO & PONTOS \\
\hline Q1 & Objetivos do estudo definidos com clareza? & Sim $=2 ;$ Parcialmente $=1 ;$ Não $=0$ & $0-2$ \\
\hline Q2 & Usuários/Participantes descritos? & Sim $=2$; Parcialmente $=1 ;$ Não $=0$ & $0-2$ \\
\hline Q3 & Materiais e métodos foram descritos? & Sim $=2 ;$ Parcialmente $=1 ;$ Não $=0$ & $0-2$ \\
\hline Q4 & A experiência com o material foi descrita? & Sim $=2 ;$ Parcialmente $=1 ;$ Não $=0$ & $0-2$ \\
\hline Q5 & A experiência do usuário foi descrita? & Sim $=2 ;$ Parcialmente $=1 ;$ Não $=0$ & $0-2$ \\
\hline Q6 & Resultados destalhados? & Sim $=2 ;$ Parcialmente $=1 ;$ Não $=0$ & $0-2$ \\
\hline Q7 & Apresentação dos dados estatísticos dos resultados? & Sim $=2 ;$ Parcialmente $=1 ;$ Não $=0$ & $0-2$ \\
\hline Q8 & Conclusões perspicazes? & Sim $=2$; Parcialmente $=1 ;$ Não $=0$ & $0-2$ \\
\hline Q9 & Critérios de sustentabilidade? & Sim $=2 ;$ Parcialmente $=1 ;$ Não $=0$ & $0-2$ \\
\hline & Somatória & & $0-18$ \\
\hline
\end{tabular}

Fonte: Elaborado pelos autors

Para a segunda etapa de análise definiram-se os critérios com base na revisão da literatura, onde se observaram métodos comuns de experimentação. No caso da experiência material (VEELAERT et al., 2020), pode-se considerar o tipo do estímulo, sendo Virtual ou Físico, e o tipo do material, em formato de Produto ou Textura. Camere e Karana (2018) definem que a experiência do usuário perante materiais variados apresenta quatro níveis de interação, podendo ser: Performativa; Sensorial; Interpretativa; e Afetiva. Ainda que os artigos analisados não utilizem especificamente o 
método proposto pelas autoras, essas características previamente definidas ajudam a delimitar os tipos de resultados encontrados. Como a qualidade Performativa está ligada a testes de usabilidade do material e isso normalmente requisita estudos mais completos, não encontrados na pesquisa, ela não foi incluída.

Quadro 4: Critérios avaliação das questões da experiência material

\begin{tabular}{|l|l|}
\hline QUESTÕES & RESPOSTAS \\
\hline Tipo do estímulo com o material & Virtual ou Físico \\
\hline Tipo do material & Produto ou Textura \\
\hline Produto & Descrição do produto quando aplicável \\
\hline Material experienciado & Nome do material \\
\hline Qualidades experimentais & Sensorial, Interpretativa, Afetiva \\
\hline & Fonte: Elaborado pelos autores \\
\hline
\end{tabular}

Em suma, Choi (2016) considera que a multiplicidade de usuários dificulta que se defina um modelo para avaliar a percepção de diferentes materiais. Nesse sentido, as metodologias propostas tendem a ser subjetivas e, portanto, as análises foram predominantemente qualitativas.

\section{Resultados e discussões}

Seguindo as etapas do método PRISMA (Figura 2), consideraram-se para a revisão integrativa e análise de dados um total de 15 publicações científicas, entre artigos de periódicos e de anais de congresso, conforme pode ser visualizado no diagrama a seguir:

Figura 2: Diagrama de etapas de pesquisa

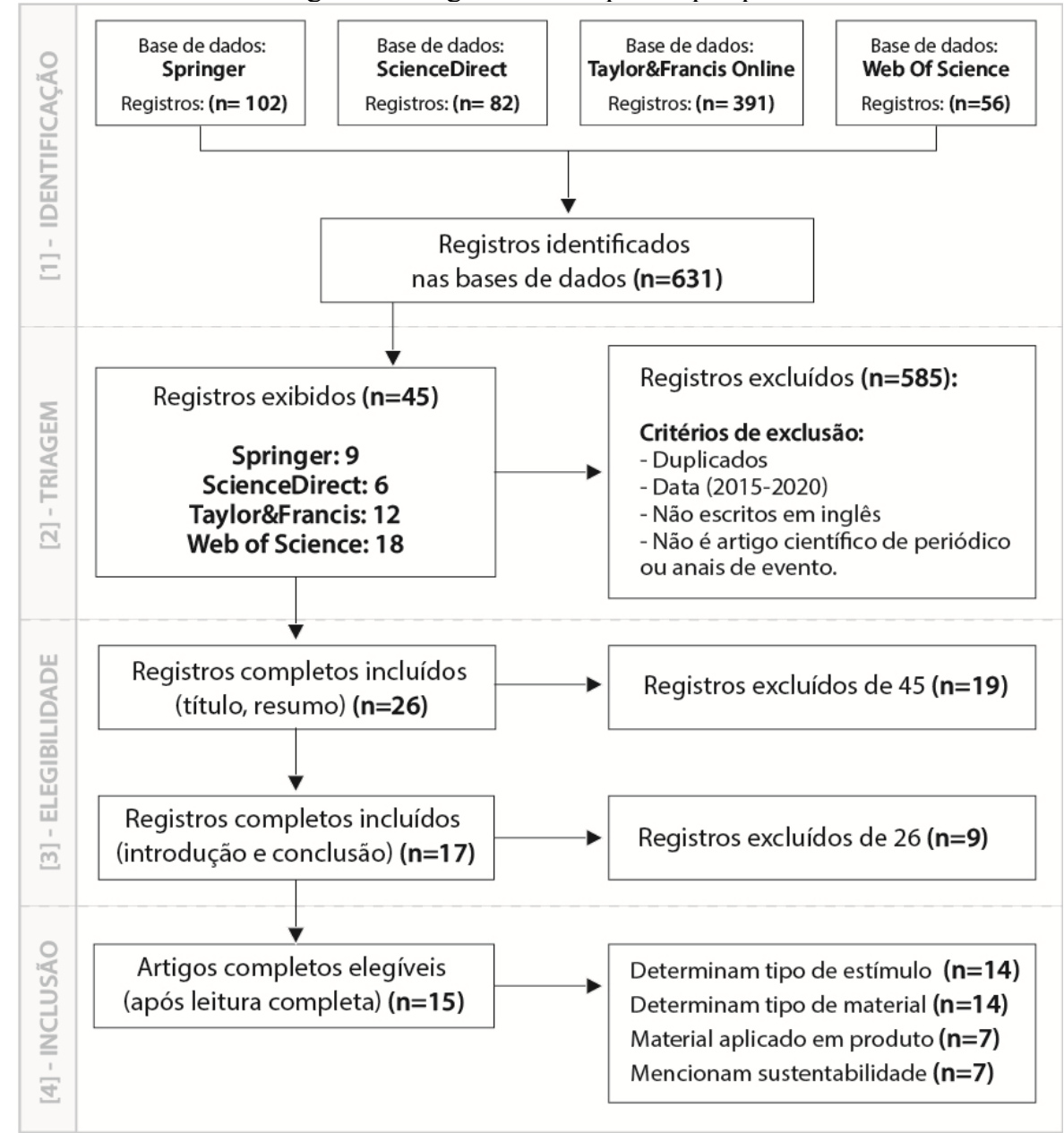

Fonte: Elaborado pelos autores 
De acordo com os resultados exibidos nos mecanismos de busca, a experiência com materiais pôde ser observada em diversos campos de conhecimento, não apenas no design. Ela foi identificada, por exemplo, em pesquisas realizadas em materiais biocompatíveis para aplicações na medicina, avaliações sensoriais em ambientes arquitetônicos; e até em análises sensoriais específicas do setor alimentício. Apesar de todas essas possibilidades se encaixarem na identificação de materiais, os respectivos artigos foram descartados da análise de resultados desta revisão. Para tanto, foram selecionados apenas aquelas pesquisas que se associassem mais diretamente com o design de produtos.

Todos os artigos eleitos para a etapa de inclusão e análise estão descritos no quadro 5 a seguir.

Quadro 5: Artigos selecionados para a revisão integrativa

\begin{tabular}{|c|c|c|}
\hline CÓD. & TÍTULO DO ARTIGO & AUTORES \\
\hline A01 & An investigation of material perception in virtual environments. & $(\mathrm{NIU}, \mathrm{LO}, 2019)$ \\
\hline A02 & Cosmetic obsolescence? & (LILLEY et al.,2016) \\
\hline $\mathbf{A 0 3}$ & Crafting Concrete as a material for enhancing meaningful interactions. & (WANG et al., 2017) \\
\hline A04 & $\begin{array}{l}\text { Design for Enhancing Material Appreciation: An Application on the Palm } \\
\text { Tree Midribs. }\end{array}$ & (ALAA, LOFTI, 2019) \\
\hline A05 & $\begin{array}{l}\text { Design for Meaningful Materials Experience: A Case Study About } \\
\text { Designing Materials with Rice and Sea-Salt }\end{array}$ & (YIN et al., 2017) \\
\hline A06 & Experiencing the Elements - User Study with Natural Material Probes & $\begin{array}{l}\text { (HAKKILA, COLLEY, } \\
\text { 2015) }\end{array}$ \\
\hline A07 & $\begin{array}{l}\text { Exploring the Fit Between Materials' Expressive Values and the Self- } \\
\text { expression of the End-User }\end{array}$ & $\begin{array}{l}\text { (VEELAERT et al., } \\
\text { 2019) }\end{array}$ \\
\hline A08 & Make the environment the (Next) Economy & (MONNA, 2017) \\
\hline A09 & Material perception and material identification in product design. & $\begin{array}{l}\text { (NDENGUE et al., } \\
2017 \text { ) }\end{array}$ \\
\hline A10 & $\begin{array}{l}\text { Material selection by the evaluation of diffuse interface ofmaterial } \\
\text { perception and product personality }\end{array}$ & (CHOI, 2016) \\
\hline A11 & $\begin{array}{l}\text { Material Tinkering. An inspirational approach for experiential leaming and } \\
\text { envisioning in product design education }\end{array}$ & (PARISI et al., 2017) \\
\hline A12 & Materials, use and contaminated interaction. & (BAXTER et al., 2015) \\
\hline $\mathbf{A 1 3}$ & Sensory Perception of Material Texture in Consumer Products & (ZUO et al., 2016) \\
\hline A14 & $\begin{array}{l}\text { The Promise of Intravaginal Rings for Prevention: User Perceptions of } \\
\text { Biomechanical Properties and Implications for Prevention Product } \\
\text { Development }\end{array}$ & (GUTHRIE et al., 2015) \\
\hline A15 & $\begin{array}{l}\text { Towards the Meaningful 3D-Printed Object: Understanding the Materiality } \\
\text { of 3D Prints }\end{array}$ & (NAM et al., 2019) \\
\hline
\end{tabular}

Fonte: Elaborado pelos autores

Destes artigos escolhidos, 9 foram encontrados no buscador Web of Science, 4 no Springer e 2 no Taylor\&Francis. Dentre as quatro bases de dados selecionadas, a ScienceDirect não foi incluída na análise final pois os artigos de interesse, que haviam sido selecionados, estavam duplicados em comparação aos encontrados em outras plataformas.

Sobre os anos das publicações, observou-se uma distribuição quase uniforme ao longo do período escolhido (de 2015 a 2020), sendo 3 deles de 2015, 3 de 2016, 5 de 2017 e 4 de 2019.

Os artigos foram lidos inicialmente tendo como base os critérios de avaliação de qualidade mencionados anteriormente (Quadro 3). Essa análise das questões encontra-se disposta na Tabela 1, onde identificou-se que as publicações cumpriram uma média de $81,10 \%$ no atendimento dos requisitos estabelecidos. Nessa fase, observaram-se os objetivos propostos em cada estudo, a descrição de seus materiais e métodos, a caracterização do processo experimental, a inclusão da análise perceptiva da experiência com usuários, a obtenção de resultados e a análise dos mesmos.

Dos 15 trabalhos avaliados, 13 deles informaram uma quantidade similar de participantes na coleta de dados, apresentando uma variação entre 11 e 38 pessoas e uma média de 22 usuários por estudo. 
Tabela 1: Avaliação dos critérios

\begin{tabular}{|l|c|c|c|c|c|c|c|c|c|c|c|}
\hline $\begin{array}{c}\text { CÓD. } \\
\text { ARTIGO }\end{array}$ & Q1 & Q2 & Q3 & Q4 & Q5 & Q6 & Q7 & Q8 & Q9 & TOTAL & $\%$ \\
\hline A01 & 2 & 2 & 2 & 2 & 2 & 2 & 2 & 2 & 0 & 16 & $88.88 \%$ \\
\hline A02 & 2 & 2 & 2 & 2 & 2 & 2 & 2 & 2 & 2 & 18 & $100.0 \%$ \\
\hline A03 & 2 & 2 & 2 & 2 & 2 & 1 & 1 & 1 & 0 & 13 & $72.22 \%$ \\
\hline A04 & 2 & 2 & 2 & 2 & 2 & 2 & 2 & 2 & 1 & 17 & $94.44 \%$ \\
\hline A05 & 2 & 0 & 2 & 1 & 1 & 1 & 1 & 1 & 0 & 9 & $50.00 \%$ \\
\hline A06 & 2 & 2 & 2 & 2 & 2 & 2 & 2 & 1 & 1 & 16 & $88.88 \%$ \\
\hline A07 & 2 & 2 & 2 & 2 & 2 & 2 & 2 & 2 & 0 & 16 & $88.88 \%$ \\
\hline A08 & 2 & 0 & 2 & 1 & 1 & 1 & 0 & 2 & 2 & 11 & $61.11 \%$ \\
\hline A09 & 1 & 1 & 2 & 1 & 2 & 1 & 1 & 1 & 1 & 11 & $61.11 \%$ \\
\hline A10 & 2 & 2 & 2 & 2 & 2 & 2 & 2 & 2 & 0 & 16 & $88.88 \%$ \\
\hline A11 & 2 & 1 & 2 & 2 & 2 & 1 & 1 & 2 & 1 & 14 & $77.77 \%$ \\
\hline A12 & 2 & 2 & 2 & 0 & 2 & 2 & 2 & 2 & 0 & 14 & $77.77 \%$ \\
\hline A13 & 2 & 2 & 2 & 2 & 2 & 2 & 2 & 2 & 1 & 17 & $94.44 \%$ \\
\hline A14 & 2 & 2 & 2 & 1 & 2 & 2 & 2 & 2 & 0 & 15 & $83.33 \%$ \\
\hline A15 & 2 & 2 & 2 & 2 & 2 & 2 & 2 & 2 & 0 & 16 & $88.88 \%$ \\
\hline MÉDIA & & & & & & & & & & & $81.10 \%$ \\
\hline TOTAL & & & & & & & & & & & \\
\hline
\end{tabular}

Fonte: Elaborado pelos autores

Tendo como base os critérios sugeridos para a avaliação da experiência do usuário com o material (Quadro 4), a análise dos artigos identificou os pontos mais relevantes da prática experimental, como as qualidades perceptivas e os diferentes materiais estudados (Quadro 6).

Quadro 6: Artigos avaliados pelos critérios da experiência do usuário com material

\begin{tabular}{|c|c|c|c|c|c|c|c|c|c|}
\hline \multirow[b]{2}{*}{$\begin{array}{l}\text { Cód. } \\
\text { Artigo }\end{array}$} & \multicolumn{2}{|c|}{$\begin{array}{l}\text { Tipo do } \\
\text { estímulo }\end{array}$} & \multicolumn{2}{|c|}{$\begin{array}{l}\text { Tipo do } \\
\text { material }\end{array}$} & \multirow[b]{2}{*}{ PRODUTO } & \multirow[b]{2}{*}{ MATERIAL } & \multicolumn{3}{|c|}{ Qualidades } \\
\hline & 恶 & $\frac{8}{\stackrel{\circ}{0}}$ & $\begin{array}{l}\stackrel{O}{\Xi} \\
\stackrel{\Xi}{\circ} \\
\text { D. }\end{array}$ & 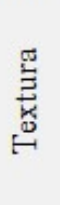 & & & 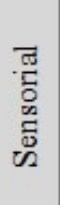 & 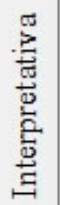 & 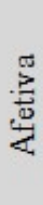 \\
\hline A01 & $\mathbf{X}$ & & $\mathbf{X}$ & & Chaleira elétrica & Aço inoxidável e plástico & $\mathbf{X}$ & & \\
\hline A02 & & $\mathbf{X}$ & $\mathbf{X}$ & & $\begin{array}{l}\text { Capinha } \\
\text { de celular }\end{array}$ & $\begin{array}{l}\text { Borracha, couro, nogueira, plástico, } \\
\text { titânio, cortiça, bambu }\end{array}$ & $\mathbf{X}$ & & \\
\hline A03 & & $\mathbf{X}$ & & $\mathbf{X}$ & - & Concreto & $\mathbf{X}$ & $\mathbf{X}$ & \\
\hline A04 & & $\mathbf{X}$ & $\mathbf{X}$ & & Mobiliário & Ripas de palmeira & $\mathbf{X}$ & $\mathbf{X}$ & $\mathbf{X}$ \\
\hline A05 & & $\mathbf{X}$ & & $\mathbf{X}$ & - & Compósito de arroz e sal marinho & $\mathbf{X}$ & $\mathbf{X}$ & $\mathbf{X}$ \\
\hline A06 & & $\mathbf{X}$ & & $\mathbf{X}$ & - & $\begin{array}{l}\text { Agua, pedra, bolhas de sabão, gelo, areia, } \\
\text { vento, fogo }\end{array}$ & $\mathbf{X}$ & $\mathbf{X}$ & $\mathbf{X}$ \\
\hline A07 & $\mathbf{X}$ & & & $\mathbf{X}$ & - & $\begin{array}{l}\text { Metais, polímeros e elastômeros, } \\
\text { cerâmica e vidro, compósitos naturais }\end{array}$ & $\mathbf{X}$ & $\mathbf{X}$ & $\mathbf{X}$ \\
\hline A08 & & $\mathbf{X}$ & & $\mathbf{X}$ & - & Compósito de micélio & $\mathbf{X}$ & $\mathbf{X}$ & $\mathbf{X}$ \\
\hline A09 & & $\mathbf{X}$ & $\mathbf{X}$ & & $\begin{array}{l}\text { Capinha } \\
\text { de celular }\end{array}$ & $\begin{array}{l}\text { Plástico com repres entação gráfica de } \\
\text { outros materiais }\end{array}$ & & $\mathbf{X}$ & $\mathbf{X}$ \\
\hline Al0 & - & - & - & - & - & $\begin{array}{l}\text { Metal, cerâmica, polímero, materiais } \\
\text { naturais }\end{array}$ & $\mathbf{X}$ & $\mathbf{X}$ & \\
\hline Al1 & & $\mathbf{X}$ & & $\mathbf{X}$ & - & Materiais experimentais & $\mathbf{X}$ & & \\
\hline A12 & & & $\mathbf{X}$ & & Variados & Não especificado & $\mathbf{X}$ & $\mathbf{X}$ & \\
\hline A13 & & $\mathbf{X}$ & $\mathbf{X}$ & & Secador cabelo & Metal, plástico, elastômeros & $\mathbf{X}$ & $\mathbf{X}$ & $\mathbf{X}$ \\
\hline Al4 & & $\mathbf{X}$ & $\mathbf{X}$ & & $\begin{array}{c}\text { Anel } \\
\text { intra-vaginal }\end{array}$ & Polímero flexível & $\mathbf{X}$ & & \\
\hline A15 & & $\mathbf{X}$ & & $\mathbf{X}$ & - & PLA, resina SLA, plástico comum & $\mathbf{X}$ & $\mathbf{X}$ & $\mathbf{X}$ \\
\hline
\end{tabular}

Fonte: Elaborado pelos autores 
Poucas publicações sugeriram o uso de estímulo virtual para se experimentar com materiais e esses, utilizaram texturas ao invés de produtos com materiais aplicados, justificando que o uso da tecnologia deve facilitar a vida do designer ao permitir mais acesso à materiais digitais e mais versatilidade na criação de modelos de simulação 3D. Apesar dessa vantagem mencionada, observouse uma predominância de estudos com práticas de experimentação focadas na percepção sensorial com o material físico. Enquanto no ambiente virtual é possível explorar uma variedade maior de texturas visuais, as publicações que exploram o objeto físico ressaltam a influência de uma experiência sensorial mais completa, por parte tanto do designer quanto do usuário, no desenvolvimento de produtos mais eficientes.

Para que o designer saiba fazer escolhas que não apenas cativem o usuário em um primeiro momento, mas que o mantenham como consumidor por um prazo maior, é preciso considerar a importância de se entender a interação do usuário com um produto/sistema. Em um dos artigos analisados, Lilley et al. (2016) observam como o desgaste dos materiais, mais especificamente capinhas de celular, causam desgosto no usuário quando apresentam sinais estéticos e físicos de uso. Quando feitas de plástico, estas capas tendem a perder, com pouco uso, algumas propriedades sensoriais como suavidade, leveza e brilho, caracterisitcas significativas no consumo de eletrônicos pois passam impressão de tecnologia e modernidade. A perda, não apenas estética, mas também de funcionalidade do material pode causar o descarte antecipado do produto e ser uma malefício para a natureza ao aumentar um consumo não sustentável a longo prazo. Uma preocupação a ser considerada diante dos questionamentos para soluções mais ecologicamente eficientes.

Por fim, foi possível identificar que, se por um lado alguns artigos tiveram por objetivo o entendimento da experiência do usuário em si (BAXTER et al., 2015; HAKKILA, COLLEY, 2015; VEELAERT et al., 2019), por outro as práticas empíricas focaram na aplicação de materiais experimentais variados (PARISI et al., 2017) como, por exemplo, compósito de micélio (MONNA, 2017), compósito de arroz e sal (YIN et al., 2017), e polímeros para impressão 3D (NAM et al., 2019). Abrindo um viés de possibilidades de investigação com novas atividades práticas para a exploração de materiais não convencionais.

\section{Considerações Finais}

O principal propóstio deste estudo foi identificar as experiências do usuário em relação ao material no contexto do design do produto, propósito atingido após a revisão sistemática integrativa realizada.

Em geral, as pesquisas analisadas identificaram como os usuários interagem com um produto/textura por meios dos sentidos, utilizando principalmente a visão e o tato. Isto representa que em experiências de cunho cognitivo e de formação de opinião, a interação estabelece uma relação psicológica que irá influenciar questões de satisfação/insatisfação do consumidor com o objeto consumido.

Observou-se que a percepção sensorial apresenta diferentes níveis de ocorrência, sendo primeiro uma relação subjetiva interpretativa e emocional do usuário, e em um segundo momento, uma relação com os aspectos do contexto de uso. O critério de performance foi muito pouco observado nos estudos avaliados, pois além de requisitar abordagens mais completas do material, costumam estar associados aos produtos em si e suas características de usabilidade, o que não foi o foco em nenhum dos artigos.

As publicações revisadas mostram uma tendência em pesquisas que investigam não apenas a experiência material do usuário final, mas também a experiência dos próprios designers que, pela interação e caracterização dos materiais, conseguem atribuir novas propriedades e realizar aplicações diferenciadas nos produtos. Essa tendência condiz com conformação do método do Material Driven Design proposto por Karana et al. (2015), que sugere a manipulação de novos materiais sustentáveis na primeira fase de um projeto de produto.

Ainda que o foco do presente estudo não tenha sido o de se identificar a experiência do usuário especificamente com materiais ecologicamente eficientes, a sustentabilidade foi sim um fator incluído como um critério de análise devido à sua importância no contexto atual de novos recursos. Entretanto, a revisão bibliográfica mostrou que a menção do mesmo foi incipiente, mesmo naqueles artigos que trataram do assunto. 
Com a revisão integrativa foi possível verificar que a pesquisa com materiais e a análise da experiência do usuário é algo recorrente dentro do design, principalmente quando se tem uma preocupação com as ferramentas/processos disponíveis e com o desenvolvimento de objetos mais ergonômicos. Nesse contexto, apesar dos resultados tenderem a ser subjetivos, por envolverem perfis de amostragem diferentes, a ocorrência de pesquisas sobre a experimentação com materiais pode contribuir para a melhora da experiência do usuário com os objetos e ajudar a consolidação de quesitos de usabilidade nos artefatos.

The experience with materials in design: An integrative review on user perception

Abstract: The user experience in product design is directly related to the perception of materials that form and consolidate an object. The way of interacting with an artifact involves questions that range from its shape and physical properties, to the implication of the user's personal and social aspects. The experience with materials explores the aesthetic, sensory and contextual characteristics of use, helping the designer to better understand the user and develop products with a more ergonomic and functional design. This can also contribute to the experimentation with new materials and to the emergence of questions associated with the sustainability of resources. In this context, the research aimed to understand how the user experience materials. Through a systematic bibliographic review, the investigation selected 15 papers from design about specific practices of experimentation with materials. The results of the analysis allowed to identify the types of stimuli used, the characteristics of the materials involved and the cognitive qualities of the user's experience.

Keywords: Design; user experience; ergonomics; perception; materials.

\section{Agradecimentos}

Este estudo obteve apoio do CNPq - Conselho Nacional de Desenvolvimento Científico e Tecnológico Processo 304619/2018-3.

\section{Referências bibliográficas}

ALAA, El Anssary; LOFTI, Nariman G. Design for Enhancing Material Appreciation: An Application on the Palm Tree Midribs. In: Materials Research Proceedings 11, 2019, pp. 315-324, 2019.

ALLAM, Hassan; HUSSIN, Razak Che; DAHLAN, Halina Mohamed. User Experience: challenges and opportunities. Journal of Research and Innovation in Information Systems, 2013.

BAXTER, Weston L.; AURISICCHIO, Marco; CHILDS, Peter R. N. Materials, use and contaminated interaction. Materials and Design, v. 90, pp. 1218-1227, 2016.

CAMERE, Serena; KARANA, Elvin. Experiential characterization of materials: Towards a toolkit. Proceedings DSR 2018 - Design Research Society International Conference 2018: Catalyst, 25-28 June, Limerick/Ireland, 2018.

CYBIS, Walter; BETIOL, Adriana Holtz; FAUST, Richard. Ergonomia e Usabilidade: Conhecimentos, Métodos e Aplicações. São Paulo: Novatec Editora Ltda, 2015.

CHOI, Jaeho. Material selection by evaluation of diffuse interface of material perception and product personality. International Journal of Interactive Design and Manufacturing (IJIDEM), v. 11, pp.967-977, 2016. 
GUTHRIE, Kate Morrow; VARGAS, Sara; SHAW, Julia G.; ROSEN, Rochelle K.; VAN DER BERG, Jacob J.; KISER, Patrick F.; et al. The Promise of Intravaginal Rings for Prevention: User Perceptions of Biomechanical Properties and Implications for Prevention Product Development. PLoS ONE 10 (12): e0145642, 2015.

HÄKKILA, Jonna; HE, Yun; COLLEY, Ashley. Experiencing the Elements - User Study with Natural Material Probes. In: 15th Human-Computer Interaction (INTERACT), Sep 2015, Bamberg, Germany. pp.324-331, 2017.

KARANA, E., BARATI, B., ROGNOLI, V., \& ZEEUW VAN DER LAAN, A. (2015). Material driven design (MDD): A method to design for material experiences. International Journal of Design, $9(2)$, 3554, 2015.

KARANA, Elvin; PEDGLEY, Owain; ROGNOLI, Valentina. On materials experience. Design Issues, v. 31, n. 13, summer, 2015.

LILLEY, D.; SMALLEY, G.; BRIDGENS, B.; WILSON, G.T.; BALASUNDARAM, K. Cosmetic obsolescence? User perception of new and artificially aged materials. Materials and Design, v. 101, pp. 355-365, 2016.

MONNA, Vanessa. Make the environment the (Next) Economy. The Design Journal, 20: sup1, S1836-S1851, 2017.

NAM, Beth; BERMAN, Alex; GARCIA, Brittany; CHU, Sharon. Towards the meaningful 3D-Printed Object: Understanding the materiality of 3D Print. In: Marcus A., Wang W. (eds) Design, User Experience, and Usability. Practice and Case Studies. HCII 2019. Lecture Notes in Computer Science, vol 11586. Springer, 2019.

NDENGUE, Jessica Dacleu; JUGANARU-MATHIEU, Mihaela; FAUCHEU, Jenny. Material perception and material identification in product design. In: Proceedings of the 21st International Conference on Engineering Design (ICED17), Vol. 8: Human Behaviour in Design, Vancouver, Canada, 2017.

NIU, Mutian; LO, Cheng-Hung. An investigation of material perception in virtual environments. In: Proceedings of AHFE 2019 - International Conference on Applied Human Factor and Ergonomics, Part of the 'Advances in Intelligent Systems and Computing', volume 973, pp. 416-426, 2019.

PARISI, Stefano; ROGNOLI, Valentina; SONNEVELD, Marieke. Material Tinkering. An inspirational approach for experiential learning and envisioning in product design education. The Design Journal, 20:sup1, S1167-S1184, 2017

PRISMA. Preferred Reporting Items for Systematic Reviews and Meta-Analyses. Disponível em:< http://www.prisma-statement.org/> Acesso em Março de 2020.

TOSI, Francesca. Design for Ergonomics. Switzerland: Springer Nature, 2020.

VEELAERT, Lore; MOONS, Ingrid; COPPIETERS, Werner; BOIS, Els du. Exploring the fit between materials' expressive values and the self-expression of the end-user. In: 20th Congress International Ergonomics Association 2018, pp. 2045-2066, 2019

VEELAERT, Lore; BOIS, Els Du; MOONS, Ingrid; KARANA, Elvin. Experiential characterization of materials in product design: A literature review. Materials \& Design, v. 190, p., 2020.

ZUO, Hengfeng; JONES, Mark; HOPE, Tony; JONES, Robin. Sensory Perception of Material Texture in Consumer Products. The Design Journal, vol. 19, issue 3, pp.405-427, 2016.

WANG, Yanan; LUO, Shijian; LIU, Shuai; LU, Yujia; HANSEN, Preben. Crafting concrete as a material for enhancing meaningful interactions. In: Proceedings of 19th International Conference HCI, Human-Computer Interaction: User Interface Design, Development and Multimodality, pp.634-644, 2017.

YIN, Liang; ZHOU, Ziyu; CHENG, Hang. Design for Meaningful Materials Experience: A Case Study About Designing Materials with Rice and Sea-Salt. In: Proceedings International Conference on Cross-Cultural Design, 9th International Conference, CCD 2017, Held as Part of HCI International, 2017. 